\title{
UNIVERSAL MINIMAL SETS ${ }^{1}$
}

\author{
ROBERT ELLIS
}

Let $(X, T)$ and $(Y, T)$ be transformation groups with the same phase group $T$. A homomorphism $f: X \rightarrow Y$ is a continuous mapping such that $(x t) f=(x f) t(x \in X, t \in T)$. A transformation group $(X, T)$ is called a minimal set if $(x T)^{-}=c l s[x t / t \in T]=X(x \in X)$. In this note it will be proved that given any abstract group $T$ there exists a minimal set $(M, T)$ with compact phase space $M$ such that any minimal set $(X, T)$ with compact $X$ is a homomorphic image of $(M, T)$. Furthermore this "universal minimal set" is unique up to an isomorphism, and given $x \in M, t \in T$ with $t \neq e$ then $x t \neq x$. For a more complete discussion of several notions involved above see [2] and $[3]$.

DEFINITION 1. The $\beta$-compactification as a transformation group. Let $T$ be a discrete group, let $\beta T$ be the $\beta$-compactification of $T$, and let $t \in T$. Then the map $s \rightarrow s t$ of $T$ into $\beta T$ is continuous and so may be extended to a map of $\beta T$ into $\beta T$. Thus each element of $T$ may be identified with a homeomorphism of $\beta T$ onto $\beta T$. Under this identification $(\beta T, T)$ becomes a transformation group.

Henceforth all transformation groups $(X, T)$ will be assumed to have compact phase spaces, $X$, and discrete phase group $T$.

Lemma 1. Let $(X, T)$ be a transformation group, let $x \in X$. Then there exists a homomorphism $f$ mapping $(\beta T, T)$ onto $\left((x T)^{-}, T\right)$.

Proof. The mapping $t \rightarrow x t(t \in T)$ of $T$ into $X$ is uniformly continuous, since $x T$ is totally bounded. Hence there exists a continuous function $f$ mapping $\beta T$ onto $(x T)^{-}$with $t f=x t \quad(t \in T)$. Hence $(t s) f$ $=(t f) s(t, s \in T)$ and so by continuity $(y s) f=(y f) s(y \in \beta T, s \in T)$. The proof is completed.

Corollary 1. Let $(X, T)$ be minimal, and let $M$ be a minimal subset of $\beta T$. Then $(X, T)$ is a homomorphic image of $(M, T)$.

DEFINITION 2. Universal minimal set associated with a group $T$. A transformation group $(M, T)$ will be called a universal minimal set associated with $T$ if $M$ is minimal and if any minimal set $(X, T)$ is a homomorphic image of $(M, T)$.

Received by the editors October 26, 1959.

1 This research was supported by the United States Air Force through the Air Force Office of Scientific Research of the Air Research and Development Command, under contract AF49(638)-569. Reproduction in whole or in part is permitted for any purpose of the United States Government. 
Corollary 1 shows that any minimal subset of $(\beta T, T)$ is a universal minimal set associated with $T$.

In order to prove that all universal minimal sets associated with a given group $T$ are isomorphic I must make use of the following theorem which is proved in [1, Lemma 5$]$.

Theorem 1. Let $(X, T)$ be a transformation group, let $(I, T)$ be the transformation group associated with a minimal right ideal $I$ of the enveloping semigroup $E(X, T)$, and let $f$ be a homomorphism of I onto I. Then $f$ is an isomorphism onto.

Lemma 2. Let $(M, T)$ be a universal minimal set, and let $I$ be a minimal right ideal in $E(M, T)$. Then $(M, T)$ and $(I, T)$ are isomorphic.

Proof. Let $x \in M$. Then the map $\pi_{x}: I \rightarrow M$ such that $p \pi_{x}=x p(p \in I)$ is a homomorphism onto. Since $(M, T)$ is universal and $(I, T)$ is minimal, there exists a homomorphism $f$ of $(M, T)$ onto $(I, T)$. Hence $\pi_{x} f$ is a homomorphism of $(I, T)$ onto $(I, T)$. By Theorem 1 this map is an isomorphism onto, whence $\pi_{x}$ is one-one. The proof is completed.

Corollary 1. Let $(M, T)$ be a universal minimal set and let $f$ be a homomorphism of $M$ into $M$. Then $f$ is an isomorphism onto.

Proof. The map $f$ is onto since $M$ is minimal. Now let $I$ be a minimal right ideal in $E(M, T)$ and let $x \in M$. Then by Lemma 2, $\pi_{x} f \pi_{x}^{-1}$ is a well defined homomorphism of $(I, T)$ onto $(I, T)$. By Theorem $1, \pi_{x} f \pi_{x}^{-1}$ is one-one, hence so is $f$.

TheOREM 2. All universal minimal sets associated with the group $T$ are isomorphic.

Proof. Let $(M, T)$ and $(N, T)$ be universal minimal sets. Then there exist homomorphisms $f, g$ of $M$ onto $N$ and $N$ onto $M$ respectively. Hence $f g$ is a homomorphism of $M$ onto $M$ which by Corollary 1 to Lemma 2 must be one-one. Hence $f$ is one-one. The proof is completed.

Definition 3. Let $(X, T)$ be a transformation group. The action of $T$ on $X$ is said to be strongly effective if given $x \in X$ and $t \in T$ with $t \neq e$, then $x t \neq x$.

In order to show that the action of $T$ on its associated universal set is strongly effective I must make use of the identification of $\beta T$ with the set of ultrafilters on $T$, see [4]. Let $u$ be an ultrafilter on $T$ and $t \in T$. Then the image of $\mathcal{u}$ under $t$ is the ultrafilter $u t$ $=[U t / U \in U]$. 
TheOREM 3. Let $(M, T)$ be the universal minimal set associated with $T$. Then the action of $T$ on $M$ is strongly effective.

Proof. Let $t \in T$ with $t \neq e$ and let $\mathfrak{u}$ be an ultrafilter on $T$. I shall show that $u t \neq u$.

Let $\mathcal{F}=[F / F \subset T$ and $F t \cap F=\phi]$. Then $[e] \in \mathcal{F}$ implies that $\mathcal{F} \neq \phi$. Furthermore if $\mathcal{F}$ is ordered by inclusion, it is inductive. Let $F$ be a maximal element of $\mathcal{F}$.

Now let $x \in T$ and suppose $x \in F t \cup F$. Then $G=F \cup\{x\}$ is not in $F$ and so $G t \cap G \neq \phi$; i.e. $(F t \cup\{x t\}) \cap(F \cup\{x\}) \neq \phi$. Since $F \in \mathcal{F}$, this means that $x t \in F$. Thus $T=F \cup F t \cup F t^{-1}$.

Since $\mathcal{U}$ is an ultrafilter on $T$, one of the sets $F, F t, F t^{-1}$ must be in $\mathcal{u}$. If $\mathfrak{u} t$ were equal to $\mathfrak{u}$, then $\mathfrak{u} t^{-1}$ would also be equal to $\mathfrak{u}$. This would imply that as soon as one of the sets $F, F t, F t^{-1}$ were in $U$ they would all be in $\mathcal{u}$. This is impossible since $F \cap F t=\phi$. The proof is completed.

Let $B(T)$ be the set of functions on $T$ to the unit interval provided with the topology of pointwise convergence. Let $f \in B(T)$ and $t \in T$. Then one may define the element $f t$ of $B(T)$ in two ways; (1) $s(f t)$ $=(t s) f(s \in T)$ and (2) $s(f t)=\left(s t^{-1}\right) f(s \in T)$. In this way one obtains two transformation groups with phase group $T$. These will be denoted $B_{1}(T), B_{2}(T)$.

THEOREm 4. Let $t, s \in T$ with $t \neq s$. Then there exist functions $f, g$ $\in B(T)$ such that

1. $f(t) \neq f(s), g(t) \neq g(s)$.

2. $f$ is an almost periodic point of $B_{1}(T)$ and $g$ is an almost periodic point of $B_{2}(T)$.

3. The range of $f=$ range of $g=$ the two element set $\{0,1\}$.

Proof. Let $(M \cdot T)$ be the universal minimal set associated with $T$ and let $x \in M$. By Theorem $3 x t \neq x s$. Since $M$ is totally disconnected [4], there exists an open-closed subset $U$ of $M$ such that $x t \in U$ and $x s \notin U$. Let $h$ be the characteristic function of $U$ and set $r f$ $=(x r) h(r \in T)$. Then $f$ clearly satisfies 1 and 3 .

To show that $f$ satisfies 2 , let $\epsilon>0$ and $s_{1}, \cdots, s_{n}$ be elements of $T$. Then

$\left(f ; s_{1}, \cdots, s_{n} ; \epsilon\right)=\left[u / u \in B(T)\right.$ and $\left.\left|s_{i} u-s_{i} f\right|<\epsilon i=1, \cdots, n\right]$

is a typical neighborhood of $f$. Let $\alpha$ be an index of $M$ such that $(a, b) \in \alpha$ implies $|a h-b h|<\epsilon$, and let $\beta$ be an index on $M$ such that $(a, b) \in \beta$ implies that $\left(a s_{i}, b s_{i}\right) \in \alpha$ for $i=1, \cdots, n$. Finally let $A=[r / r \in T$ and $(x r, x) \in \beta]$. Then $A$ is a syndetic [3] subset of $T$, and 
$r \in A$ implies that $\left|s_{i}(f r)-s_{i} f\right|=\left|\left(r s_{i}\right) f-s_{i} f\right|=\left|\left(x r s_{i}\right) h-\left(x s_{i}\right) h\right|$ $<\epsilon, i=1, \cdots, n$ since $(x r, x) \in \beta$. Consequently $f r \in\left(f ; s_{i}, \cdots, s_{n} ; \epsilon\right)$ $(r \in A)$. This completes the proof for $B_{1}(T)$.

To obtain $g$, one replaces the minimal set $M$ in the above argument by the universal minimal set associated with the group $T^{*}$ opposite to $T$ (i.e. $T^{*}$ is $T$ provided with the group operation "o" where $s \circ t$ $=t s(t, s \in T))$.

Theorem 4 states that the points of any group $T$ may be separated by almost periodic points of $B(T)$. This is in marked contrast to the situation which prevails if one demands that the points of $T$ be separated by almost periodic functions in the sense of von Neumann. There exist groups $T[5]$ on which the only almost periodic functions are the constants.

\section{REFERENCES}

1. Robert Ellis, $A$ semigroup associated with a transformation group, Trans. Amer. Math. Soc. vol. 94 (1960) pp. 272-281.

2. Robert Ellis and W. H. Gottschalk, Homomorphisms of transformation groups, Trans. Amer. Math. Soc. vol. 94 (1960) pp. 258-271.

3. W. H. Gottschalk and G. A. Hedlund, Topological dynamics, Amer. Math. Soc. Colloquium Publications, vol. 36, 1955.

4. Pierre Samuel, Ultrafilters and compactification of uniform spaces, Trans. Amer. Math. Soc. vol. 64 (1948) pp. 100-132.

5. J. von Neumann, Almost periodic functions in a group. I, Trans. Amer. Math. Soc. vol. 36 (1934) pp. 445-492.

University of Pennsylvania 